\title{
Some Biological and Behavioral Studies of the Tetrastichus sp. (Hymenoptera: Eulophidae), a Pupal Parasitoid of Brontispa longissima Gestro (Coleoptera: Chrysomelidae)
}

\author{
Vivencio C. Gallego ${ }^{1}$, Ma. Cynthia E. Gallego ${ }^{2}$ and Ivorie J. dela Torre ${ }^{3}$
}

\begin{abstract}
The Tetrastichus sp. was identified as a pupal parasitoid indigenous to the Philippines. The total development period of Tetrastichus sp. from egg to adult was 16 days under laboratory conditions. The average incubation, larval and pupal periods were 3.5, 4.8, and 7.4 days, respectively. The average adult longevity was 5.5 (male) and 6.4 (female). Upon emergence of an adult wasp from the host pupa, mating took place shortly and the female look for suitable host pupa of Brontispa longissima for oviposition. Tetrastichus sp. is an endogregarious parasitoid. From one parasitized pupa of Brontispa longissima, around 6 - 66 individual wasps emerged with a mean of 22.4 while parasitized pupae collected from the field yielded $4-21$ individuals with a mean of 12.7. Majority of the wasps emerged in the morning (70\%) while 30\% emerged in the afternoon. Sex ratio of male and female was 1:5.4 in laboratory condition while 1:3.7 under field condition with females outnumbered the males. Tetrastichus sp. was observed in some cases to reproduce parthenogenetically. Longevity of Tetrastichus sp. can be enhanced to a mean of 7.3 and 6.0 days by feeding on sugar and honey, respectively while water and coconut pollen resulted in longevity of 3.3 and 2.4 days, respectively. Adults without food lived only one day. Parasitism efficiency ratio of 10 parasitoids: 10 host pupae resulted in $90 \%$ parasitism. One to two day old pupa of B. longissima was preferred by Tetrastichus sp. for oviposition with a mean of $86.7 \%$ parasitism. Percent pupal parasitism in the field ranged 25.7 $42.3 \%$ with an average of $27.6 \%$ in selected areas in Mindanao. Tetrastichus sp. was found to effectively control $B$. longissima under field condition. The study indicated that Tetrastichus $\mathrm{sp}$. is an effective control agent of $B$. longissima and could be used in the field to prevent economic damage of the palms by the pest.
\end{abstract}

Keywords: Behavior, biology, biological control, Brontispa longissima, Parasitism, Tetrastichus sp.

\footnotetext{
${ }^{1}$ Scientist I, ${ }^{2}$ Division Chief III and ${ }^{3}$ Research Assistant, Crop Protection Division, Philippine Coconut Authority - Davao Research Center, Bago-Oshiro, Davao City, Philippines.

Phone: (082)-293-0115, Fax: (082)-293-0571, E-mail: pcadrc@ pldtdsl.net.
} 


\section{Introduction}

Coconut leaf beetle, Brontispa longissima Gestro was originally described in 1885 from the Aru Islands (Maluku Province) attacking coconut palms (Cocos nucifera, L.) and but also several other cultivated and wild palms. The pest is native to Indonesia and Papua New Guinea. Over the last 126 years it has spread widely in over 25 countries in Asia, Australia and Pacific Islands (Singh and Rethinam, 2005). It may have entered the Philippines as early as 2003 but it was confirmed in 2005. The pest was spreading and the threat to coconut industry of the country was alarming.

Philippines is one of the world's top exporters and second producer of coconut products. Nearly 3.5 million coconut farmers and about 25 million people benefit from the coconut industry. Coconut is planted in 3.5 million hectares, $23 \%$ of the total croplands. This crop is one of the top 5 dollar earners (U.S. $\$ 760 \mathrm{M}$ p.a.) contributes $1.14 \%$ of the Gross National Product (GNP).

Brontispa longissima became a serious pest in the Philippines attacking bearing and non-bearing coconut. Adults and larvae feed on the lamina of young unopened fronds of coconut and other palm species. The symptoms are prominent when the affected leaves become scorched or ragged in appearance and can easily be identified even from a distance.

Two years after initial infestation action was undertaken by the Philippine Coconut Authority - Davao Research Center to determine the natural enemies of the pest. Regular field collections in different infested areas in Region XI revealed a pupal parasitoid of $B$. longissima. The specimens were sent to a taxonomist at the University of the Philippines at Los Baňos for identification. It was identified as a Tetrastichus species and the species is being investigated. This is believed to be Tetrastichus brontispae (Ferriere), native to Java, Indonesia described as the most effective natural enemy parasitizing the larval and pupal stages of the pest. It was widely introduced in the Pacific Islands and other countries for the control of Brontispa species. In addition, three egg parasitoids, three larval and pupal parasitoids, two entomopathogenic fungi and two predators were reported as natural enemies of Brontispa longissima (Singh and Rethinam, 2005). Among the parasitoids, Tetrastichus sp. is the available parasitoid in the Philippines. However, its biology and behavior have not been studied.

This study is aimed to study some aspects of the biology and behavior of the Tetrastichus sp., identify the suitable pupal stage of Brontispa longissima for oviposition by Tetrastichus sp. and to determine parasitism rates of Tetrastichus $\mathrm{sp}$. in the laboratory and field conditions.

\section{Materials and methods}

\section{Biology and Behavior of Tetrastichus sp.}

a. Rearing of Brontispa longissima and its pupal parasitoid under laboratory condition

Brontispa infested coconut spear leaves were collected from the field, sorted the eggs, larvae, healthy pupae, parasitized pupae and adults. Eggs and larvae were placed in plastic containers (18.5 x $15.5 \times 6.5$ inches) provided with fresh young coconut leaves as food. The leaves were changed every three days to avoid development of molds. The healthy pupae were used for parasitization by Tetrastichus sp. while the parasitized pupae were placed in vials for emergence of the parasitoid to be used in parasitization process as means of mass production of the Tetrastichus sp.

Adult beetles were placed in round culture plastic jars (4 in diam x 6.5 in ht) for egg deposition to continue the life cycle and to be used for biological and behavioral studies of the Tetrastichus sp. at $28{ }^{\circ} \mathrm{C}$ and $52 \%$ relative humidity.

\section{b. Life cycle of Tetrastichus sp. under laboratory condition}

Brontispa longissima, as host of Tetrastichus sp. was cultured in laboratory condition with room temperature of $28{ }^{\circ} \mathrm{C}$ and $52 \%$ relative humidity. One hundred twenty pupae of $B$. longissima that are one day old were collected from the fourth instar larvae of $B$. longissima cultures. The pupae were subjected to 
parasitization for 24 hours. The parasitized pupae were dissected for observation of the stages of Tetrastichus sp. with the aid of an stereomicroscope.

Thirty B. longissima parasitized pupae each for egg, larva, pupa and adult stages were dissected to closely monitor the developmental stages of the Tetrastichus sp. The number of days was recorded for the incubation period, larval stages and pupal stage. The adult life span was likewise monitored.

\section{c. Comparison of emergence of Tetrastichus sp. from parasitized hosts in the laboratory and field}

Thirty parasitized pupae of $B$. longissima each from the laboratory culture and field collection were placed individually in glass tubes (6" length x 1" diam.) at $28{ }^{\circ} \mathrm{C}$ temperature and $52 \%$ of relative humidity. The number of adult wasp emergence from each pupa was recorded. The Brontispa pupa cadaver was dissected under a stereomicroscope and the number of dead Tetrastichus sp. was likewise counted and recorded.

\section{d. Time of emergence and the number of exit holes made by Tetrastichus sp. adults}

Each of 30 parasitized pupae were placed individually in test tubes covered with nylon cloth and secured with rubber band. The adult wasps emerging in the morning were monitored starting 12:01 A.M. to 12:00 noon, while those in the afternoon were monitored starting at 12:01 P.M to 12:00 Midnight. The time of emergence and number of exit holes were recorded.

\section{e. Body measurements of male and female Tetrastichus sp.}

The body length of 30 male and 30 female parasitoids were measured under a stereomicroscope with the aid of a micrometer.

\section{f. Longevity of adult Tetrastichus sp. reared on different sources of food}

Longevity of adult Tetrastichus sp. was tested on honey, sugar, water, coconut pollen and no food. Very fine streaks and granules of food sources were smeared/ placed on a coconut leaflet (1' x 4" size) and placed inside the test tube (6" length $\times 1$ " diameter) together with the wasps. Thirty wasps of a day old were placed in each test tube along with the food. The number of dead wasps were counted daily and were removed from the test tubes every after observation.

\section{Parthenogenetic reproduction of adult Tetrastichus sp. under laboratory conditions}
a. Immobilizing of Tetrastichus sp. for identification and sexing

Immobilizing the parasitoid was done in the laboratory to facilitate identification and sexing. The method used by Gallego (1982) was adopted. This was done by exposing the parasitoid adults inside a test tube to cold temperature ranging from 5 to $10^{\circ} \mathrm{C}$ for $1-2$ minutes. Identification of sexes of the wasps was done after 1-2 minutes of cold exposure.

\section{b. Progeny reproduction by mated and unmated female Tetrastichus sp.}

Soon after emergence, the parasitoids were individually transferred into the test tubes without allowing mating.

Thirty tubes, each was provided with one unmated female wasp and one pupa of $B$. longissima (1-2 day old). Another thirty tubes, each was provided with a male and a female wasp and one Brontispa pupa. All test tubes with adult wasps were provided with honey as food. Wasps were kept for parasitization for 24 hours and then the parasitoid pupa was transferred individually into an individual vial for further observation. Upon emergence of the parasitoids, the number of male and female progenies in the two treatments (with mated female and unmated female) were counted under a stereomicroscope. 


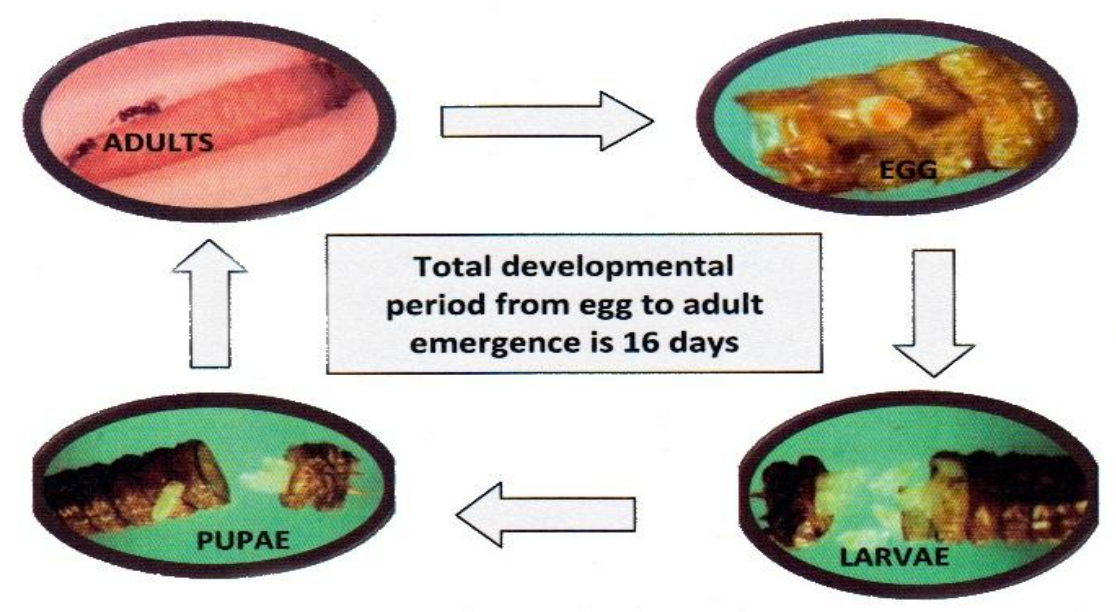

Figure 1. Life cycle of Tetrastichus sp.

\section{Effectiveness of Tetrastichus sp. in the laboratory and field condition}

\section{a. Oviposition preference of Tetrastichus sp.}

Under laboratory condition at $28{ }^{\circ} \mathrm{C}$ and $52 \%$ relative humidity, four larval stages (1st to 4th larval instars) and a pupal stage (1 day old) were introduced to Tetrastichus sp. adults to test for oviposition preference. A ratio of 10:10 parasitoids (5 males and 5 females) and host stadia of the $B$. longissima, in a plastic cup ( 2 in diam $x 2$ in ht) were used for parasitization for 24 hours and thereafter, the host stadium was removed and placed individually in a vial for further observation. Preference was based on the number and percentage parasitism, mutilation or feeding and those that developed into adult $B$. longissima using thirty hosts of each stage.

\section{b. Suitable age of Brontispa pupa for parasitization by Tetrastichus sp.}

Thirty each of, 1 day, 2 day, 3 day, 4 day and 5 day old pupae were selected from the laboratory culture. Each pupal stage (ages 1, 2, 3, 4, 5 day old) was subjected to parasitization by adult Tetrastichus sp. at the ratio of 10:10 wasps (5 males and 5 females) and pupal stage, respectively. After 24 hours of introduction or exposure for oviposition, the adult wasps of Tetrastichus sp. were removed. The number and percentage parasitism, mutilation or feeding and those that developed into adult $B$. longissima were reckoned.

\section{c. Percentage pupal parasitism by Tetrastichus sp. under field conditions}

Regular field collections were done to check natural parasitism in the field. The field collected $B$. longissima infested leaves were examined for presence of Brontispa eggs, larvae, pupae and adults. The pupal population and its parasitism were recorded. Percentage parasitism on pupae due to Tetrastichus was based on the following computation, the total number of parasitized pupae collected divided by the total number of pupae collected (healthy and parasitized) and multiplied by 100 .

\section{Results and discussion}

\section{Biology and Behavior of Tetrastichus sp.}

a. Life cycle of Tetrastichus sp. under laboratory condition

Upon emergence of the adult wasps, mating took place immediately and soon after the female wasp laid eggs by penetrating its ovipositor onto the pupa of the B. longissima (Fig.1).

The average incubation period, larval stage and pupal stage of Tetrastichus sp. was $3.5 \pm 0.7$ days which ranged from 3 to 5 days, $4.8 \pm 0.7$ days (4 to 6 days), $7.4 \pm 1.3$ days ( 4 to 9 days), 
respectively. The adult life span was 2 to 11 days with a mean of $5.5 \pm 2.6$ days for male and $6.4 \pm$ 2.8 for female. The total developmental stage from egg deposition to adult emergence was 16.0 \pm 1.3 days with a range of 12 to 19 days.

\section{b. Number of wasps emerged from laboratory cultured and field collected parasitized pupae}

Results showed that around 6 to 66 (mean of $22.40 \pm 13.54$ ) adults emerged from a single host pupa from a laboratory culture, while 4 to 21 (mean of $12.73 \pm 4.38$ ) emerged from the field collected parasitized host pupa. Higher number of emergence was expected in the laboratory culture because of a forced parasitization process done in the laboratory.

Mortality of adult wasps inside the host body in both conditions at emergence were monitored and compared. Parasitoids reared in the laboratory resulted to 6 mortalities $(1 \pm 1)$ while 3 mortalities $(0.2 \pm 1)$ from the field collected parasitized pupae. Higher mortality was observed in the laboratory culture probably due to the competition for food in the host pupa. The developing larvae are forced to pupate and developed into adult wasp but very weak and died before it emerged as adult wasp. This observation was supported by Gallego (1982) where he reported that the cause of premature mortality of hymenopteran parasitoids of Promecotheca cumingii Baly was due to competition of food from its host. Furthermore, he stated that the parasitoids that emerged were very weak and died even before feeding.

It was not possible to ascertain dead egg or larva inside the host body as it may have decomposed before the observation period.

\section{c. Time of emergence of Tetrastichus sp. and number of exit holes on host pupa}

It was noted that majority emerged in the morning with $70 \%$ emergence as early as 5:45 to 11:39 A.M. While $30 \%$ of the Tetrastichus sp. emerged in the afternoon from 2:20 PM to 9:46 in the evening. Majority emerged during day time as they are attracted to light. The parasitoid that is about to emerge, exerted its effort to create hole as its exit point. They used their antennae, forelegs, hindlegs, and ovipositor to cut an opening. Once an opening was made, adult wasp started to come out one after the other.

The number of exit holes created was dependent on the number of wasps that emerged per pupae (Fig. 2). The higher the number of wasps, the more exit holes were created. It ranged from 1 to 5 holes with an average of 2.0 \pm 1.70 .

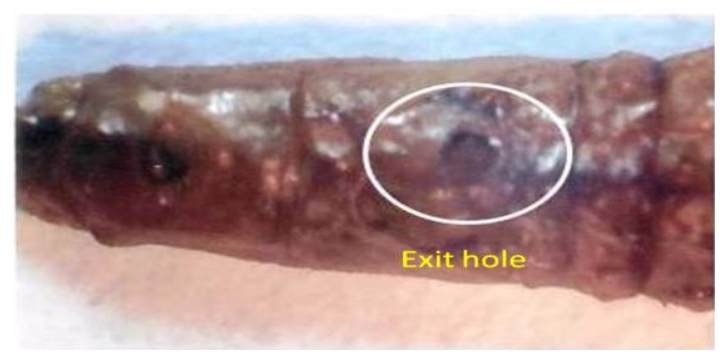

Figure 2. Brontispa pupa showing the exit hole of the Tetrastichus sp.

\section{d. Body measurements and other morphological characteristics of male and female Tetrastichus sp.}

A difference in length $(\mu \mathrm{m})$ of male and female wasps was noted. The female wasps were longer than the males with an average size of $6.01 \pm 0.92$ and $5.29 \pm 0.80 \mu \mathrm{m}$, respectively.

Other morphological characteristic to identify male and female Tetrastichus sp. was through their distinct antennae (Fig. 3). The female antennae were colored pale yellow in the half of the antenna from base of the head and the other half to the tip were black. The male antennae were all black.
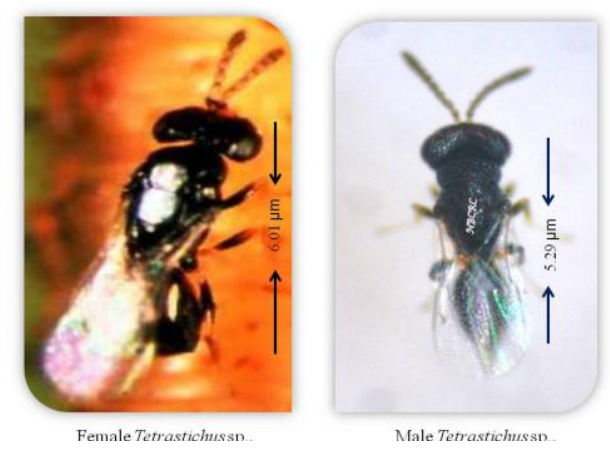

Figure 3. Female (left) and male (right) Tetrastichus sp. 
Table 1. Mean longevity (days) of adult Tetrastichus sp. after exposure to different food sources

\begin{tabular}{|l|c|c|}
\hline \multicolumn{1}{|c|}{ Food source } & Range & Mean* \\
\hline Sugar & $2-15$ & $7.3^{\mathrm{a}}$ \\
Honey & $1-9$ & $6.0^{\mathrm{a}}$ \\
Water & $1-4$ & $3.3^{\mathrm{b}}$ \\
Coconut Pollen & $2-3$ & $2.4^{\mathrm{cb}}$ \\
No food & 1 & $1.0^{\mathrm{c}}$ \\
\hline
\end{tabular}

* Means with the same letter(s) are not significantly different at 5\% level DMRT.

Table 2. Sex ratio of Tetrastichus sp.

\begin{tabular}{|l|c|c|}
\hline \multicolumn{1}{|c|}{ Sex } & Range & Mean \\
\hline $\begin{array}{l}\text { Fertilized female Tetrastichus sp. } \\
\text { Male }\end{array}$ & $1-3$ & $1.47 \pm 0.6$ \\
Female Ratio (M:F) & $3-15$ & $8.00 \pm 3.0$ \\
\multicolumn{1}{|c|}{} & & $1: 5.4$ \\
\hline Un-fertilized female Tetrastichus sp. & $2-15$ & $9.0 \pm 3.05$ \\
Male Female & 0 & 0 \\
\hline
\end{tabular}

Table 3. Preference stage of Tetrastichus sp. for oviposition

\begin{tabular}{|l|c|c|}
\hline \multicolumn{1}{|c|}{ Stages } & \% Parasitism */ & \% Mutilation / Feeding */ \\
\hline $1^{\text {st }}$ Instar larvae & $0^{\mathrm{c}}$ & $46.7^{\mathrm{ab}}$ \\
$2^{\text {nd }}$ Instar Larvae & $0^{\mathrm{c}}$ & $60.0^{\mathrm{a}}$ \\
$3^{\text {rd }}$ Instar Larvae & $0^{\mathrm{c}}$ & $66.7^{\mathrm{a}}$ \\
$4^{\text {th }}$ Instar Larvae & $16.7^{\mathrm{b}}$ & $30.0^{\mathrm{ab}}$ \\
Pupal stage & $90.0^{\mathrm{a}}$ & $6.7^{\mathrm{b}}$ \\
\hline
\end{tabular}

*/ Means with the same letter(s) are not significantly different at 5\% level DMRT. 


\section{e. Longevity of adult Tetrastichus sp. on different food sources}

Sugar was the most preferred food for the adult wasps of Tetrastichus sp. with a resulting longevity of 7.3 days (Table 1). This was significantly higher compared with the other alternate foods evaluated.

\section{Parthenogenetic reproduction of adult Tetrastichus sp. under laboratory condition}

As shown in Table 2, the mated females produced progenies of both males and females with mean of 1.47 and 8.0, respectively. The sex ratio was recorded at 1:5.4 (M:F). On the otherhand, unmated females produced all male progenies.

III. Effectiveness of Tetrastichus sp. in laboratory and field conditions against Brontispa longissima

\section{a. Oviposition preference of Tetrastichus sp.}

As shown in Table 3, the pupal stage of the $B$. longissima was the most preferred host for the oviposition of the adult wasps. The percentage parasitism was significantly higher on pupae compared to the other host stages evaluated. The $4^{\text {th }}$ instar larval stage of the $B$. longissima had $16.7 \%$ parasitism, while the $1^{\text {st }}$, $2^{\text {nd }}$ and $3^{\text {rd }}$ larval stages resulted in zero parasitism. Hence, the pupal stage of $B$. longissima can be used for the mass production of Tetrastichus sp. in the laboratory.

The low parasitism on the last instar larvae indicated that it is not the preferred stage for parasitism and it may have been due to the unavailability of suitable stage for Tetrastichus sp. oviposition. Mutilation or host feeding by Tetrastichus sp. on $1^{\text {st }}, 2^{\text {nd }}, 3^{\text {rd }}$ and $4^{\text {th }}$ instar larvae were observed under laboratory conditions with percentage of 46.7, 60.0, 66.7 and $30.0 \%$, respectively. These could as well add to the host mortality in field conditions.

\section{b. Suitable age of Brontispa pupae for parasitism by Tetrastichus $s p$.}

It was observed that 1 - 4 day old pupae were the best ages of parasitization by Tetrastichus sp. The percentage parasitism of pupae decreased with the age of the pupae (Table 4).
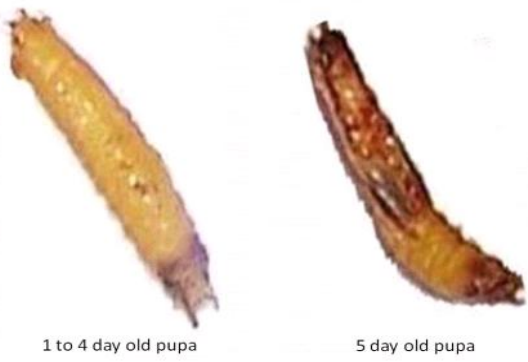

Debach and White (1960) demonstrated the ability of a parasitoid to kill more hosts by frequent insertions of its ovipositor shaft without oviposition, referred to as mutilation which was also observed by Thompson and Hagen (1999) that probing and host mutilation as well as feeding on early instar larvae contributed to host mortality.

As stated further by Sandanaayake and Edirisinghe (1992), although oviposition in the early instar larvae could be thought of as a wasteful phenomenon, since it does not lead to parasitoid development, it could still be considered as a good characteristic of a parasitoid since it would lead to host mortality.

Table 4. Suitable pupal age by Tetrastichus sp. for oviposition

\begin{tabular}{|c|c|c|}
\hline $\begin{array}{c}\text { Pupal stage } \\
\text { (days) }\end{array}$ & $\begin{array}{c}\text { Percentage } \\
\text { Parasitism* }\end{array}$ & $\begin{array}{c}\text { Percentage } \\
\text { Mutilation / } \\
\text { Feeding* }\end{array}$ \\
\hline 1 & $86.7^{\mathrm{a}}$ & $13.0^{\mathrm{b}}$ \\
2 & $86.7^{\mathrm{a}}$ & $13.0^{\mathrm{b}}$ \\
3 & $83.3^{\mathrm{a}}$ & $10.0^{\mathrm{b}}$ \\
4 & $60.0^{\mathrm{a}}$ & $26.7^{\mathrm{b}}$ \\
5 & $23.3^{\mathrm{b}}$ & $63.3^{\mathrm{a}}$ \\
\hline
\end{tabular}

*Means with same letter(s) are not significantly different at 5\% level DMRT 


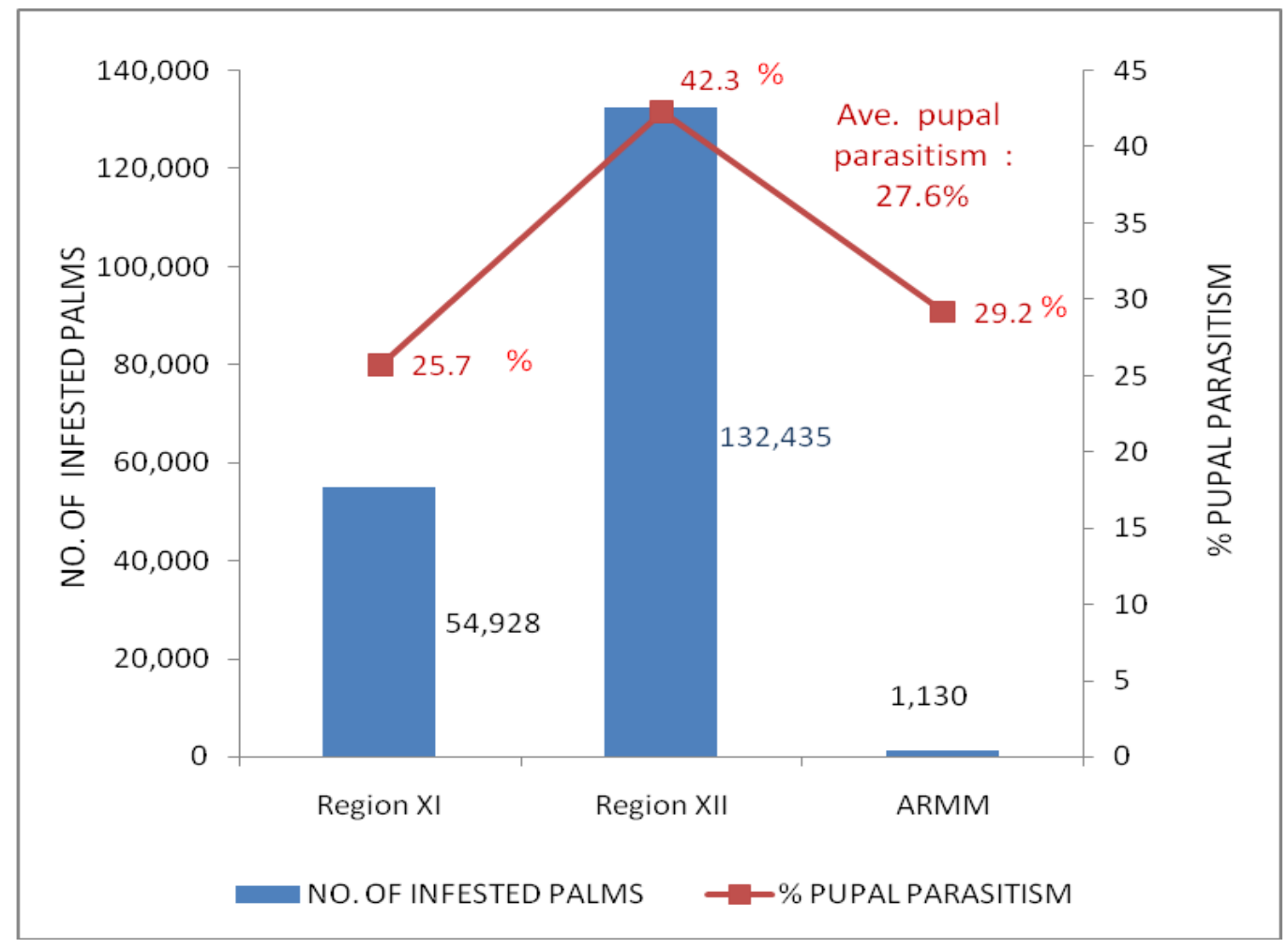

Figure 4. Number of Brontispa infested palms and percent parasitism by Tetrastichus sp.
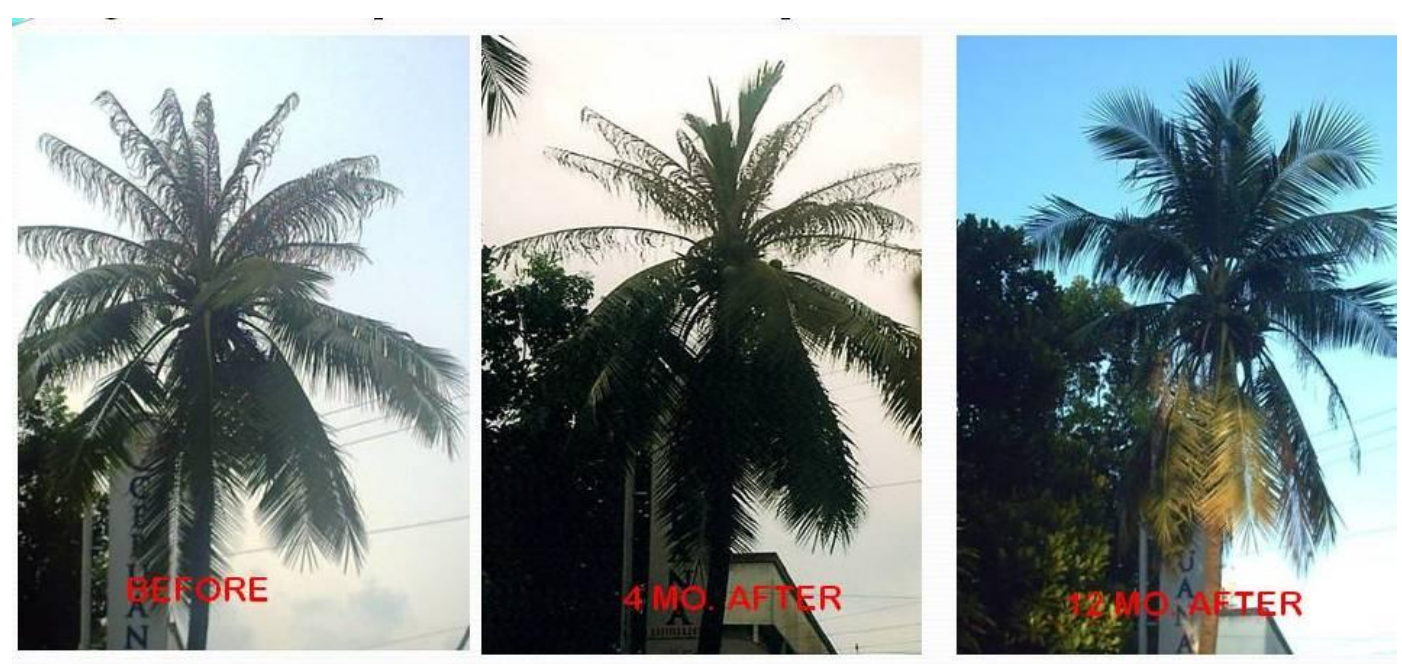

- Brontispa-infested palm released with pupal parasitoids showed new undamaged emerging fronds after four and 12 months

Figure 5. Effect of Tetrastichus sp. released on damaged palms 


\section{c. Percentage pupal parasitism by the Tetrastichus sp. under field conditions}

The parasitized pupae were dark colored, inflated and mummified, an indication that the host was killed by the parasitization. Under laboratory conditions, these marks were observed from 4 days oviposition of the Tetrastichus sp. adult female. The unparasitized pupae were yellowish-white in color.

The initial level of field parasitism by Tetrastichus sp. from different sampling areas in Mindanao resulted $25.7 \%, 42.3 \%$ and $29.2 \%$ parasitism in Region XI, Region XII and ARMM, respectively with an average of $27.6 \%$ which was quite high in the absence of any augmentative releases of the parasitoid (Fig. 5). It was apparent that the Tetrastichus sp. was indigenous to these areas.

In Figure 5, the Brontispa-infested palm released with Tetrastichus__sp. showed undamaged emerging leaves after four months and fully recovered after a year, indicating the effectiveness of releasing the parasitoids.

Tetrastichus sp., therefore has the potential to regulate $B$. longissima populations and prevent an economic damage to coconut palm. It is therefore recommended that mass production of this parasitoid and inundative releases be undertaken to increase the level of parasitism in the field.

In addition, it is likewise recommended to plant leguminous crops and/ or other flowering plants under coconut to increase food sources for the adult parasitoids to feed. As stated by Gallego (1982), the parasitoids in proximity to their source of food such as the flowering plants around the coconut plantation at emergence can contribute to the increase in level of the parasitoid population.

\section{Conclusion}

The study revealed that Tetrastichus sp. is a pupal parasitoid that is indigenous to the Philippines. Being a gregarious parasitoid with high parasitism levels, it had the potential to control B. longissima Gestro in the country.
Furthermore, results of the studies are very important and provide vital information necessary in the formulation of IPM strategies to manage $B$. longissima population in the field below economic level.

\section{Acknowledgement}

The authors would like to acknowledge the funding support of Philippine Coconut Authority (PCA)-Central Office, Diliman, Quezon City, Department of Agriculture (HVCC, AFMA), Department of Science and Technology (DOST) and Philippine Council for Agriculture, Aquaculture and Natural Resources Research and Development (PCAARRD). We also thank the management and staff of PCADavao Research Center for the administrative and financial support services. Appreciation is also extended to Southern Mindanao Agriculture and Resources Research and Development Consortium (SMARRDEC) for the Annual Scientific Conferences to showcase the output of the research.

\section{References}

Debach, P. and White, E.B. 1960. Commercial mass culture of the California red scale parasite, Aphytis lingnanensis. Univ. Calif. Agr. Expt. Sta. Bull., 770 p.

Gallego, V.C. 1982. Some Ecological Studies of the Coconut Leafminer (Promecotheca cumingii, Baly) and its Hymenopterous Parasitoids in the Philippines. Masters Thesis. UPLB.

Thompson, S.N. and Hagen, K.S. 1999. Patterns of host feeding by hymenopteran parasitoids. Handbook of Biological Control. Academic Press. 606 p.

Sandanaayake, W.R.M. and Edirisinghe, J.P. 1992. Trathala flavoorbitalis and its application. 13(3):287-292.

Singh, S.P. and Rethinam, P. 2005. Coconut leaf beetle (Brontispa longissima). Asia and Pacific Coconut Community JakartaIndonesia. 